occur in the cellar. We took one specimen of what appeared to be this species from under the bark of a dead maple tree which would indicate that it is possibly indigenous. It is reported from New York by Mr. Macgillivray (Canad. Ent. Dec. r89i, p. 272) but under what conditions is not mentioned.

Remarks. The meagre description given by Lubbock in his monograph permits us to make but few comparisons of the characters of our specimens with $S$. buskii, yet though our specimens differ in the shorter, basal, antennal joint and broader band between the eyes, we see no reason for separating our specimens, for they agree exactly in form and other color markings.

Seira purpurea Schött, Beitrage zur Kenntniss Kalifornischen Collembola, Bihang Kongl. Svensk. vet. akad. handl. bd. 17 afd. iv, no. 8, p. I7, Pl. IV. (fig. I). Flava, signaturis purpureis quae in segmento thoracico tertio et in segmento abdominis primo secundoque fascias, totam aream segmenti non occupantes, formant. Segmenta abdominalia cetera purpurea. Long. 2-I, $5 \mathrm{~mm}$.

Da die Gattungen Entomobrya Rondani und Sira Lubbock sich nur durch das Vorhandensein oder Nichtvorhandensein von Schuppen unterscheiden, so ist es natürlich unmöglich die Gattung der hierhergehörenden Formen zu bestimmen, da nur in Weingeist conserviertes Material vorliegt. Wenn ich aber nun obige Form zur ersten
Gattung führe, so geschieht dies nur wegen der Zeichnung derselben. Diese erinnert insofern an diejenige bei unseren Sira-arten, dass keine Fascien mit scharf markierten Konturem wie bei allen bisher bekannten zweifarbigen Entomobyra-formen vorhanden sind, sondern nur langgestreckte Flecke, die one scharfe Begrenzung in die Grundfarbe übergehen.

Die Form ist ausnehmend schön und von den schwedischen Arten der Gattung Sira Lubbock wohl unterschieden. Das erste Thoracalsegment ist rotviolett, das zweite oben ganz hell, hat aber an der Kante einen dunklen Rand, welcher von dem ganz. dunkel gefärbten Basalglied des zweiten Extremitätenpaares, das wie die übrigen dunkle Cozalglieder hat, nach der entgegengesetzten Seite verläuft. Das dritte Thoracalsegment und die beiden ersten $A b d o m i-$ nalsegmente sind ungefähr gleich und mit violetten Querbändern versehen, die sich nur über die Dorsalfläche der Segmente erstrecken, wohingegen die Seiten gelbweiss sind. Das dritte und vierte Abdominalsegment sind völlig violett, das fünfte und sechste von gemischtem Colorit. Das Manubrium der Gabel und ein kleinerer teil der Dentes sind schwach violett, der übrige Teil und die Endsegmente ungefärbt. Der Kopf ist hell, die Augen stehen auf schwarzen Elecken mit einer dunklen Verbindungslinie zwischen sich. Die Antennenglieder sind schwach violett, heller nach den Spitzen hin. Die Abdominalsegmente, besonders die hintern, sind mit dichten Haaren versehen, die noch dichter auf den beiden Seiten sind und sich nach den Dentes furculae fortsetzen, auch in der Nackenregion befinden sich solche Haare.

\section{A NEW FAMILY OF FOSSIL BEETLES.}

In a recent paper on the fossil insects of Rott on the Rhine (Abh. naturf. ges. Halle, $\mathrm{xx}$ ) Schlechtendahl establishes a new family
Palaeogyrinidae on a beetle showing a combination of the characters of Gyrinidae and Dytiscidae. Extinct types of insects of as high a grade as families are extremely rare in the tertiaries. 

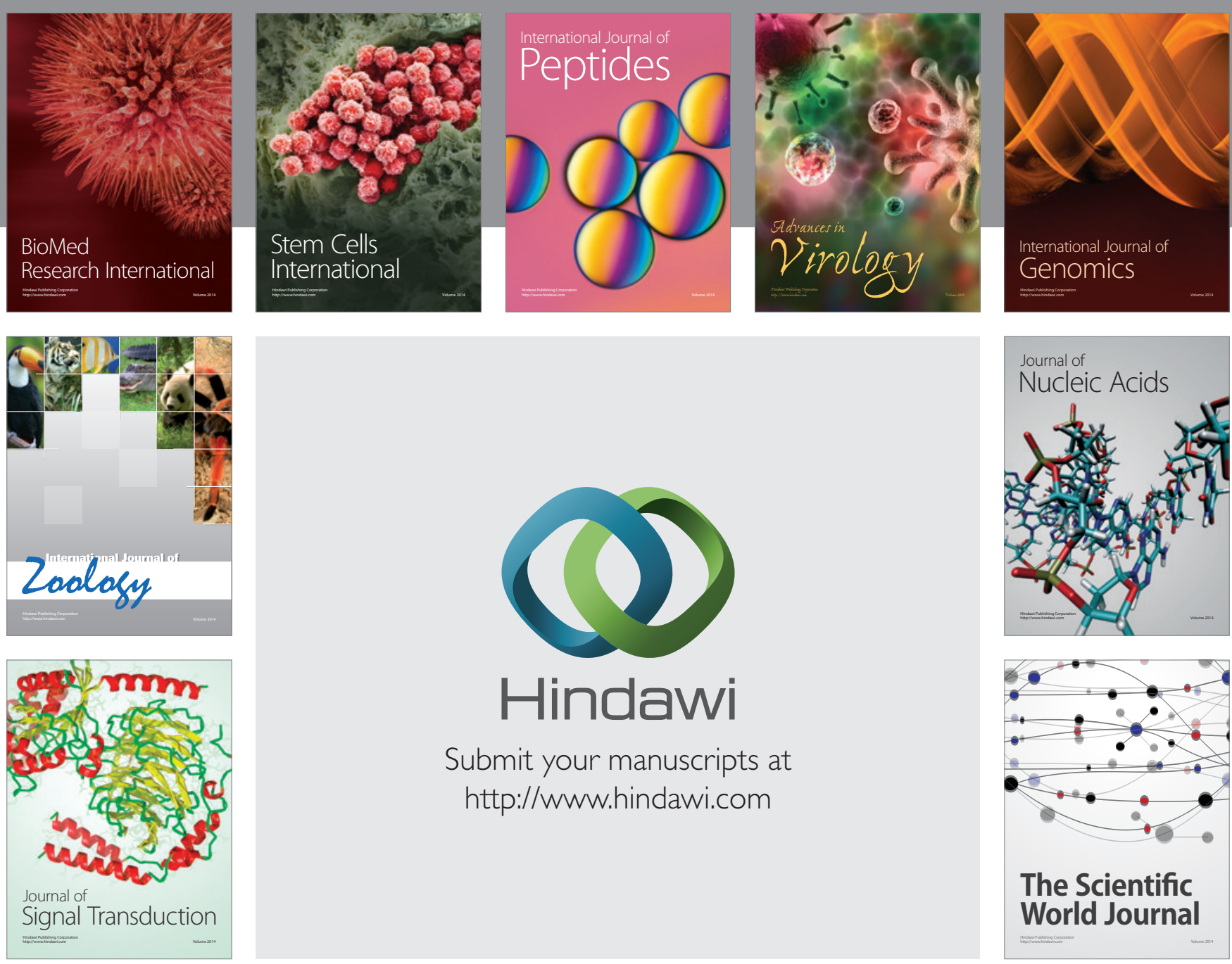

Submit your manuscripts at

http://www.hindawi.com
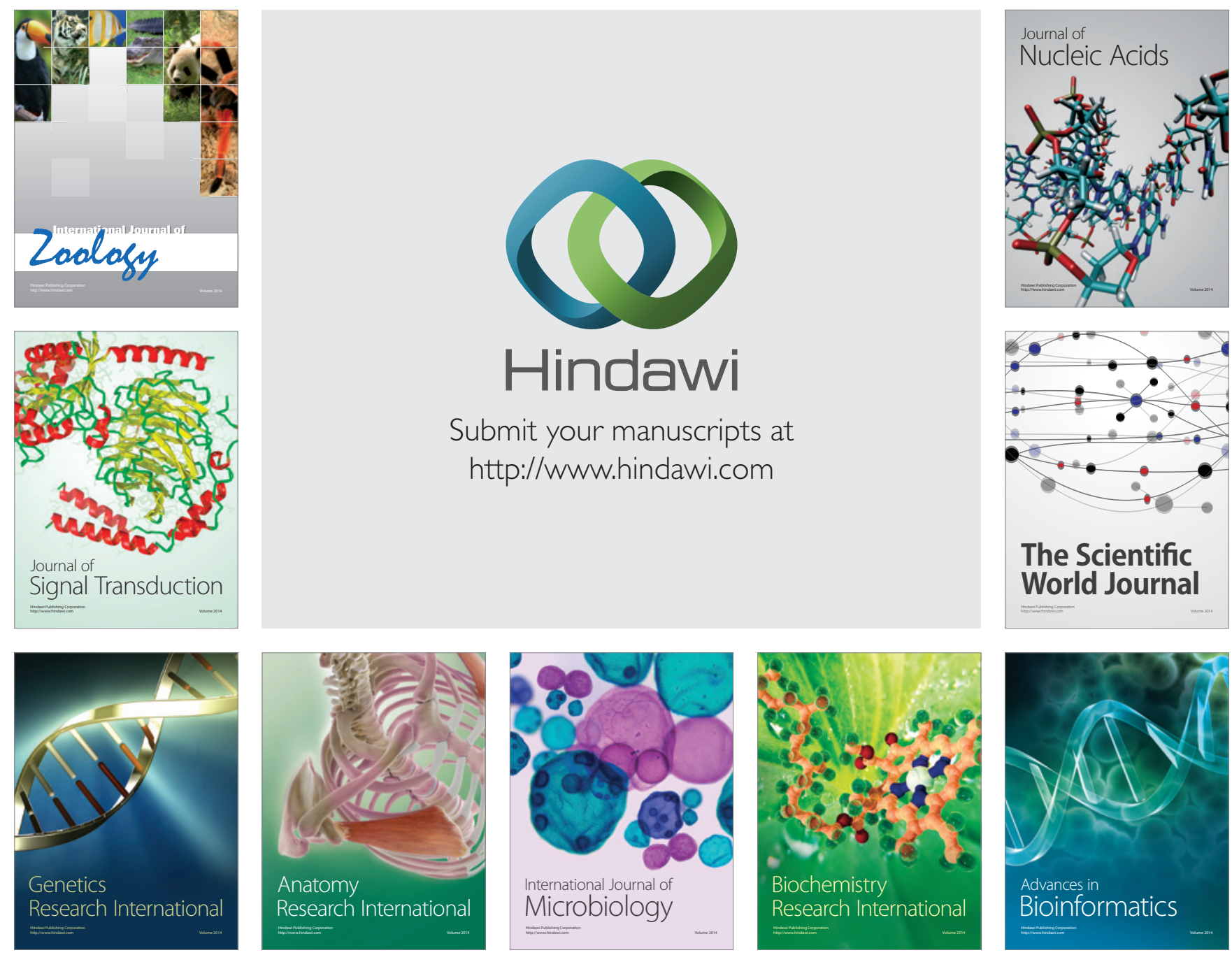

The Scientific World Journal
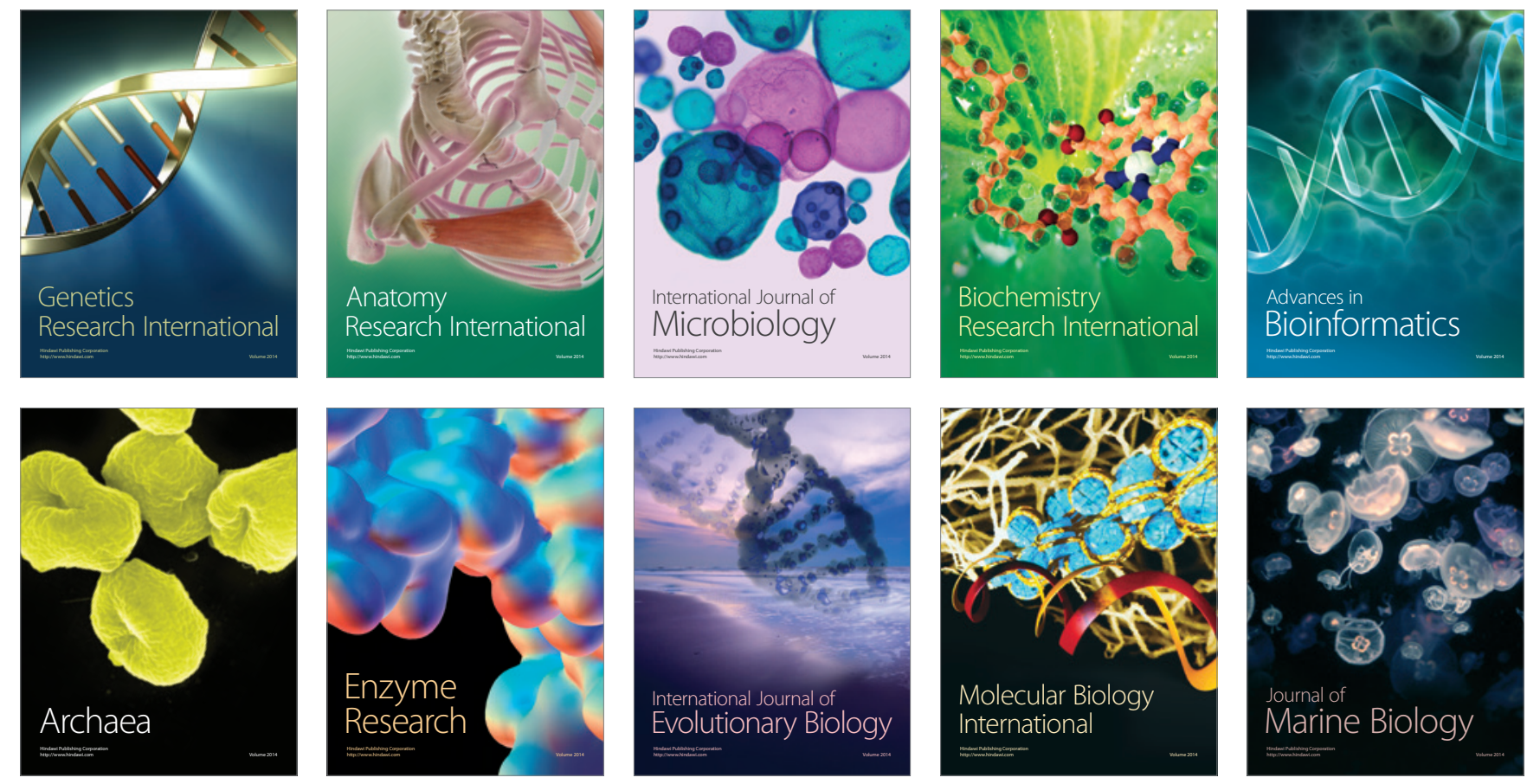